\title{
Ultrafast gating using a nonlinear Sagnac interferometer
}

\author{
François Salin, Jeff Squier and Kathryn Clay \\ Center for Ultrafast Optical Science, University of Michigan, 2200 Bonisteel, Ann Arbor MI-48109-2099, USA
}

Received 5 August 1991; revised manuscript received 6 November 1991

\begin{abstract}
We show theoretically and experimentally that Sagnac interferometers which include a medium which exhibits gain or absorption can be used to obtain picosecond or femtosecond optical gates with very high contrast and good transmission. This method is well suited for imaging through scattering media or extracting a weak signal from a broad background.
\end{abstract}

Ultrafast gating is a powerful technique for applications such as time resolved spectroscopy [1-3], imaging through scattering media [4-7], or nonlinear optics [8]. Several methods have been used, each of which has its own limitations. The Kerr shutter [3] is extremely fast (femtosecond) but requires high peak power lasers. It has a maximum transmission of approximately 10 percent, and induces a strong phase modulation on the signal. The streak camera [8] has a temporal resolution of a few picoseconds and is not capable of 2D time resolved imaging. Parametric amplification sampling spectroscopy [2] provides gain (up to $10^{4}$ ) with femtosecond resolution. In its simplest version the amplified signal is surrounded by a large background which for applications such as imaging through scattering media is not acceptable. The traveling-wave amplifier [9] also provides a very high gain (up to $10^{9}$ ) with a 100 femtosecond gating window but produces an output signal even in the absence of an input signal. We present here a method based on a Sagnac interferometer. The technique is capable of ultrafast (femtosecond) gating with a very high contrast (up to $10^{4}$ ) and large amplification ratio of the signal. Although it also has its own limitations, it is well suited for imaging through scattering media and in particular for medical imaging.

The principle of the method is to induce a non-reciprocal transmission change in an antiresonant-ring or Sagnac interferometer. The Sagnac interferometer $[10,11]$ is a modified version of the Michelson interferometer in which both beams travel exactly along the same path (fig. 1). The important feature of this interferometer is that the intensity of the light leaving the ring (i.e. not reflected back to the input) is determined by the intensity of the two beams traveling in the ring and not by their coherence properties. For example, it is insensitive to length drift. The intensity of the leakage is given by

$I_{\text {out }}=\left|E_{1}-E_{2}\right|^{2}$,

where $E_{1}$ and $E_{2}$ are the two electric fields counterpropagating in the ring. When the beam splitter has a $50 \%$ reflectivity then $I_{\text {out }}=0$. If we introduce an element which can change the amplitude or phase transmission between the time of arrival of the first beam and that of the second beam, $E_{1}$ no longer equals $E_{2}$ when they recombine on the beam splitter and $I_{\text {out }} \neq 0$. Such leakage can be obtained through several different effects [10-15]. It has most recently been used in an antiresonant-ring transient spectroscopy experiment [16] by Trebino and Hayden. They have shown that, by placing a sample in an antiresonant ring and by exciting it with an ultrashort pulse, one can enhance the signal to noise ratio of pump and probe experiments. They also noticed that by placing the sample off center in the ring they could suppress signals from long time scale (picosecond or longer) phenomena. The method presented here, which we call Sagnac interferometer gating (SIG), has been inspired by these results but differs by the fact that we are not interested in the properties of the sample itself but we do however, want to use them to gate an external signal. Ultrafast 


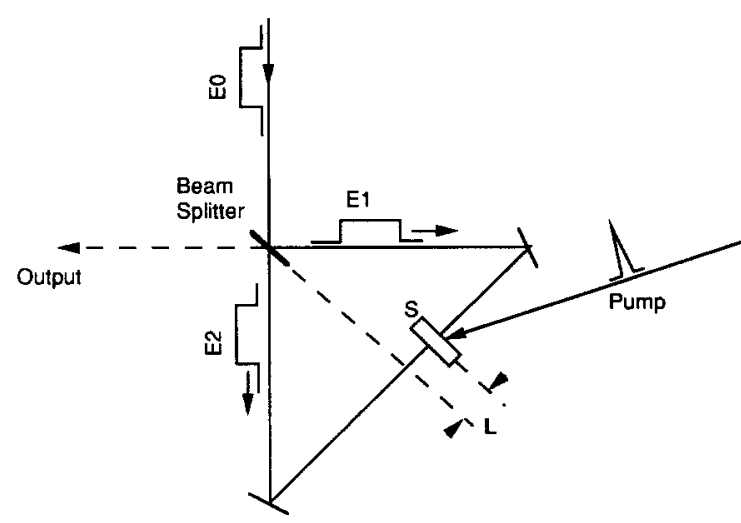

Fig. 1. Sagnac interferometer used for ultrafast gating. The input is a long pulse which is split into two replicas by the beam splitter. The sample $S$ is placed at a distance $L$ from the center of the ring. The pump is a short pulse capable of changing the sample properties. The output is the beam which is not reflected back to the input.

gating has been proposed by $\mathrm{Li}$ and co-workers [15], using the Kerr effect in a Sagnac interferometer. Their method used classical interferometry instead of polarization interferometry but otherwise was equivalent to the Kerr shutter and suffered the same drawbacks.

The schematic of the experiment is given in fig. 1. A sample which can be a saturable absorber, an optical amplifier (based on laser or parametric effects) or any nonlinear component which properties can be changed instantaneously, is placed at a distance $L$ from the center of a Sagnac interferometer. The sample can be excited by an ultrashort laser pulse (pump pulse). We will limit the discussion to amplitude change in the sample although phase change can be used as well [15]. A pulse much longer than the pump pulse is incident on the ring. Ideally, the beam splitter reflectivity is $50 \%$. We will look at the leakage signal leaving the ring through the beam splitter (see fig. 1). In absence of excitation both pulses 1 and 2 suffer the same loss or gain and no light leaks through the beam splitter. If the sample is excited by an ultrashort pulse an instantaneous change in the amplitude of both pulses occurs. As the sample is not at the center of the ring, this transition does not occur at the same instant for both pulses. Therefore, the two pulses exiting the ring will have different temporal shapes and cannot perfectly subtract which

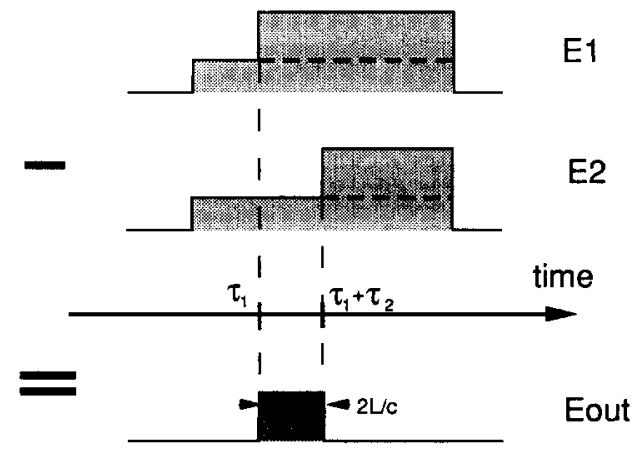

Fig. 2. Gating principle. The sample is initially absorbant. The dotted line corresponds to the pulse shapes incident on the beam splitter if the sample is unpumped. In that case the output is null. The solid line corresponds to the pulse shapes after passing through the pumped sample. The pump arrival corresponds to time $t_{1}$ for pulse 1 and time $t_{1}+t_{2}$ for pulse 2 . The output is the difference between pulse 1 and pulse 2 . It consists of a pulse of duration $t_{2}=2 L / c$.

results in leakage. In fig. 2 we took the example of a long square pulse and of a saturable absorber which is bleached by a much shorter pulse. The two first lines show pulses 1 and 2 before and after they go through the sample. The time delay between the transmission change on pulse 1 and pulse 2 corresponds to $2 L / c$ where $c$ is the light velocity and $L$ is the distance from the sample to the center of the ring. The beam splitter acts as a optical subtracter and the signal leaking from the ring is a short pulse of duration $2 L / c$. Note that this duration does not depend on the properties of the sample as long as its recovery time is much longer than the incident pulses. Different instants of the incident signal can be gated by adjusting the delay between the pump and the signal.

The intensity leaking from the beam splitter can be written as

$$
\begin{aligned}
& I_{\text {out }}=\mid R E_{0}(t) T\left(t+\tau_{1}\right) \\
& \quad-\left.(1-R) E_{0}(t) T\left(t+\tau_{1}+\tau_{2}\right)\right|^{2},
\end{aligned}
$$

where $R$ is the reflectivity of the beam splitter, $E_{0}$ is the signal electric field, $T(t)$ is the time dependent transmission of the sample, $\tau_{1}$ is the arrival time of the pump pulse and $\tau_{2}=2 L / c$. Depending on the physical effect which is used in order to induce the leak, $T(t)$ can have different forms. We studied three 
types of effects: saturable absorption, laser amplification and parametric amplification.

The saturable absorber transmission depends on the integrated energy absorbed by the medium. When the relaxation time of the excited state is much longer than any of the pulses the transmission can be written as

$T_{\mathrm{a}}(t)=\exp \left(-\frac{\gamma_{0}}{1+s(t)}\right)$,

where $\gamma_{0}$ is the unbleached absorption and $s(t)$ is the integrated fluence of the pump normalized to the saturation fluence $J_{\text {sat }}$ of the saturable absorber:

$S(t)=\frac{1}{J_{\mathrm{sat}}} \int_{-\infty}^{t} I\left(t^{\prime}\right) \mathrm{d} t^{\prime}$,

with $I(t)$ the pump pulse intensity.

Fig. 3 shows the transmission $I_{\text {out }} / I_{\text {in }}$ of the Sagnac interferometer for different distances $L$. The sample is excited by a hyperbolic secant square pulse of duration $t_{\mathrm{p}}=1 \mathrm{ps}$. One can see that the gate shape evolves from a gaussian-like to a square-like shape when $L \gg c t_{\mathrm{p}}$. The maximum transmission for a long gate is given by the difference between the bleached and the unbleached transmission of the sample and therefore by the saturation parameter $s_{\max }=s(\infty)$. The maximum transmission $T_{\max }$ of the ring can be written as

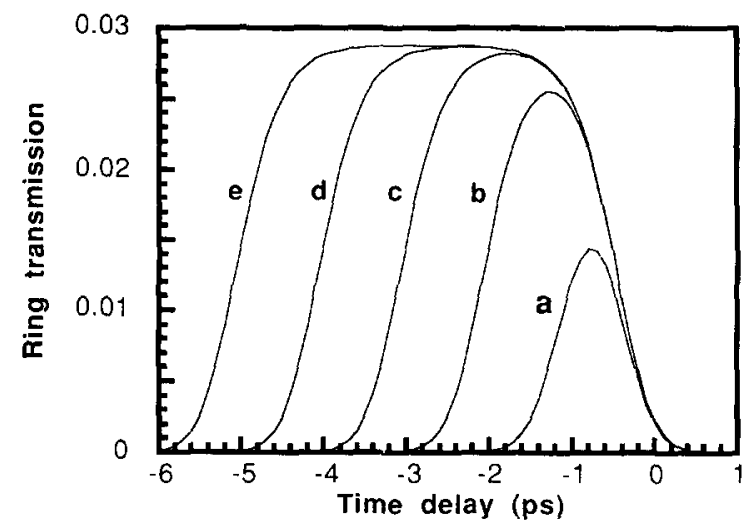

Fig. 3. Transmission of the interferometer $\left(I_{\text {out }} / I_{\text {in }}\right)$ for different positions of the sample. The sample is a saturable absorber with a small signal absorption of 0.99 . The pump pulse duration is 1 ps and the maximum pump fluence is $2 J_{\text {sat }}$ (a) $L=300 \mu \mathrm{m}$, (b) $L=600 \mu \mathrm{m}$, (c) $L=900 \mu \mathrm{m}$, (d) $L=1.2 \mathrm{~mm}$, (e) $L=1.5 \mathrm{~mm}$.
$T_{\max }=\frac{1}{2}\left[\exp \left(-\frac{\gamma_{0}}{2\left(1+s_{\max }\right)}\right)-\exp \left(-\frac{\gamma_{0}}{2}\right)\right]^{2}$

With a pump fluence equal to the saturation fluence of the absorber $\left(s_{\max }=1\right)$ and a small signal absorption $\gamma_{0}=0.99$, a maximum transmission of only $1.5 \%$ can be achieved.

As in the case of the saturable absorber, the transmission of a laser amplifier depends on the integrated energy absorbed by the gain medium. It can be written as

$T_{\mathrm{g}}(t)=\exp [s(t)]$,

where $s(t)$ is given by expression (3) in which $J_{\text {sat }}$ is the gain saturation fluence at the signal wavelength. Fig. 4 shows the transmission of a Sagnac interferometer including a gain medium for different distance $L$. Because the gain is an exponential function of the pump fluence, the gate obtained with a gain medium is slightly shorter than that obtained with a saturable absorber under the same conditions. The maximum transmission achievable is given by

$T_{\max }=\frac{1}{2}\left[\exp \left(\frac{1}{2} J_{\text {pump }} / J_{\text {sat }}\right)-1\right]^{2}$.

Transmission ratios much higher than 1 can be obtained using high gain laser media such as dyes.

In the case of parametric amplification, the gain is instantaneous and depends only on the pump peak

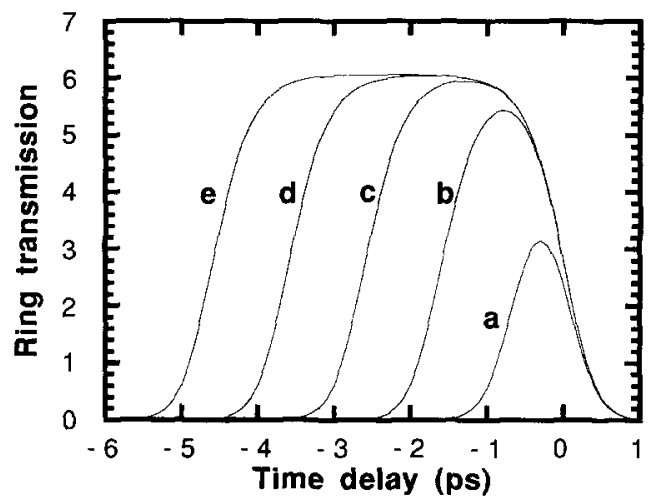

Fig. 4. Transmission of the interferometer $\left(I_{\text {out }} / I_{\text {in }}\right)$ for different positions of the sample. The sample is a laser gain medium with a small signal gain of 20 . The pump pulse duration is 1 ps. (a) $L=300 \mu \mathrm{m}$, (b) $L=600 \mu \mathrm{m}$, (c) $L=900 \mu \mathrm{m}$, (d) $L=1.2 \mathrm{~mm}$, (e) $L=1.5 \mathrm{~mm}$. 
power. It follows that, if the amplifier is placed close to the center of the ring, it will amplify a portion of the tail of pulse 1 and a portion of the front of pulse 2. After subtraction on the beam splitter, only these two amplified portions remain. For an instantaneous amplifier the gate consists of two windows separated by a delay $2 L / C$ and with the same duration as the pump pulse. This shape corresponds to the derivative of the shape obtained in fig. 4 . In order to obtain a single window the distance $L$ has to be longer than the signal pulse spatial extension $t_{\mathrm{s}} c$ where $t_{\mathrm{s}}$ is the signal pulse duration. Fig. 5 shows an example of the gate temporal shape when $L$ is smaller and much longer than $t_{\mathrm{s}} c$. Since the gain of a parametric amplifier goes as the exponential of the square of the pump intensity (neglecting any dispersing or walk-off effect) the gate duration is shorter than the pump pulse. The maximum transmission of the ring is given by

$T_{\max }=\frac{1}{2}(\sqrt{g}-1)^{2}$,

where $g$ is the peak gain of the amplifier. Single-pass gains higher than $10^{4}$ have been obtained using femtosecond pump pulses [2].

From these calculations it appears that gain media (laser or parametric) are particularly well suited for fast gating of weak signals since, not only is the background removed, but the signal is amplified. The only

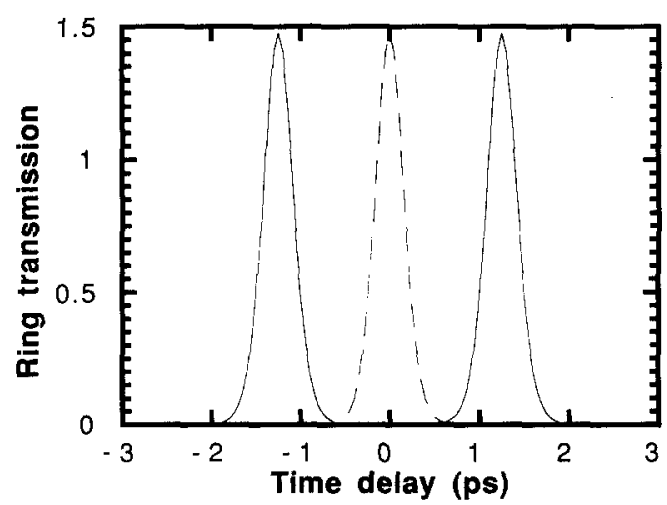

Fig. 5. Transmission of the interferometer including a parametric amplifier pumped by a 1 ps pulse. (a) The sample is placed close to the center of the ring ( $L=c t_{\mathrm{s}} / 3$ ) (solid line). (b) The sample is placed far from the center of the ring $\left(L=10 \mathrm{ct}_{\mathrm{s}}\right.$ ) (dotted line ). $t_{\mathrm{s}}$ is the signal pulse duration ( $t_{\mathrm{s}}=50 \mathrm{ps}$ in this figure). The exact position of the gate is adjusted by changing the delay between the pump and pulse 1 . drawback is that the pump pulse wavelength has to be shorter than that of the signal. One solution is to use the fundamental wavelength of an ultrashort pulse laser to create the signal and its second harmonic to pump the amplifier. With typical dyes having saturation fluences of $\sim 1 \mathrm{~mJ} / \mathrm{cm}^{2}$, gains higher than 1000 can be obtained over spot sizes of a few millimeters using a $1 \mathrm{~mJ}$ pump pulse. Note that laser gain depends only on the pump fluence and not on its peak power. The SIG technique including a laser gain medium can easily scale down in energy to accommodate high repetition rate femtosecond oscillators. Using the second harmonic of a high average power self-modelocked Ti:sapphire oscillator, a gain of $\sim 2-3$ should be obtained in highly efficient near infrared dyes. Parametric amplification, although limited to high peak power pump pulses, seems even more promising since it is capable of very high gains which, in turn, translates to higher transmission of the Sagnac interferometer. Note that since a parametric amplifier transmits all the pump light which is not transferred to the signal, one can combine a parametric amplifier and a laser amplifier in the same ring. Different methods using Raman shifting and Raman amplification or parametric oscillation can also be imagined.

We tested the viability of the SIG method using the simplest but least powerful technique: saturable absorption. The experimental set-up is given in fig. 6. The Sagnac interferometer was formed by a $50 \%$ beam splitter and two mirrors. A $2 \mathrm{~mm}$ Schott RG830 filter was placed close to the center of the ring. The pump and signal pulses were produced by a self-modelocked Ti:sapphire oscillator followed by a Ti:sapphire regenerative amplifier [17]. This source produces $8 \mathrm{~mJ}, 100 \mathrm{fs}$ pulses at $769 \mathrm{~nm}$ and at a 20 $\mathrm{Hz}$ repetition rate. In order to avoid strong nonlinear effects and spectral continuum generation induced by the pump pulse in the optics we reduced the pulse energy and lengthened its duration to $\sim 300$ fs. $80 \%$ of the incident pulse was used for the pump and the remaining $20 \%$ was apertured down to $\sim 2 \mathrm{~mm}$ and sent into the interferometer. The pump beam was slightly focused to a $\sim 2 \mathrm{~mm}$ beam on the filter. The delay between the pump and the signal pulses was adjusted using a delay line. A photodiode was used to measure the interferometer transmission. A scattering screen was placed in front of the 


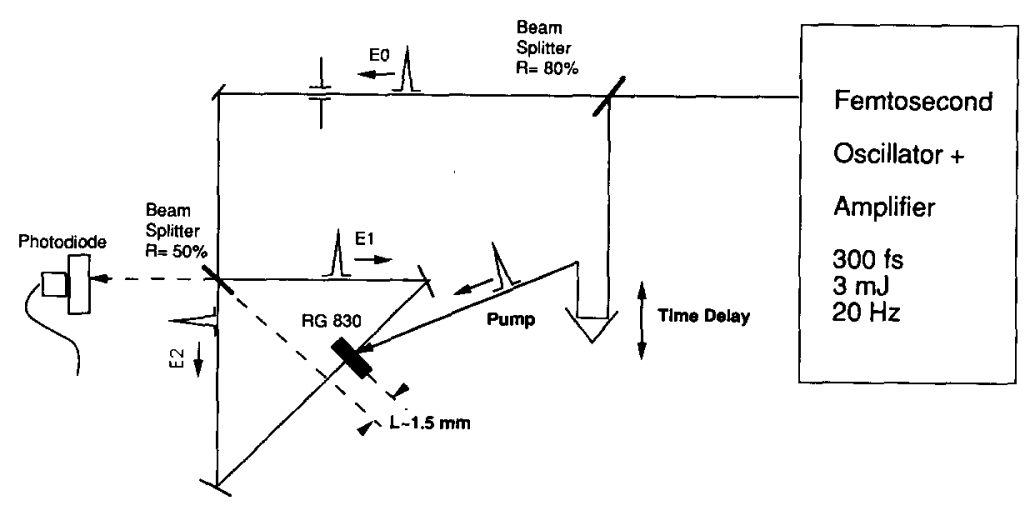

Fig. 6. Experimental set-up. The pump and signal pulse duration is $300 \mathrm{fs}$. The sample is a $2 \mathrm{~mm}$ thick, RG830 filter.

photodiode to limit the effect of beam wandering or beam inhomogeneities introduced by the sample. Since the rise time of the photodiode was much longer ( $\sim 1 \mathrm{~ns})$ than the signal duration $(300 \mathrm{fs})$ it detected the temporally integrated signal leaking through the beam splitter. Fig. 7 shows the intensity of this signal as a function of the delay between the pump and the signal. The function corresponds to the correlation of the gate shape (as shown in fig. 3) with the signal pulse shape. As a consequence the rising and falling time of the function plot in fig. 7 are longer than that of the actual gate. The ring transmission is not symmetrical. This is due to spatial in-

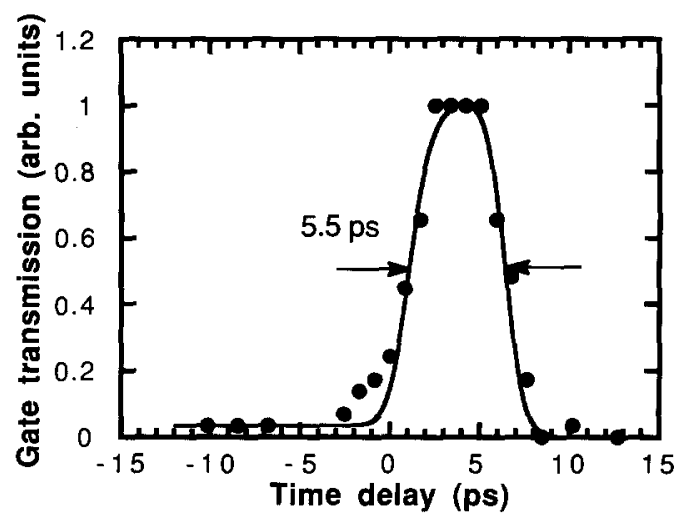

Fig. 7. Signal recorded by a photodiode looking at the leakage from the interferometer (filled dots). The gate duration has been arbitrarily chosen. The solid line is a fit assuming a $1.2 \mathrm{ps}$ pump pulse and $2 \%$ leakage due to spatial inhomogeneities introduced by the sample. homogeneities introduced by the poor optical quantity of the sample. When the pump pulse arrives in the sample after both pulses 1 and 2 (delay $>+10$ ps), these pulses are absorbed and even if the interferometer is not perfectly aligned no light will be transmitted. When the pump pulse arrives in the sample before the signal pulses, the saturation is bleached and any misalignment or spatial imperfection (wedge, non-perfect flatness, ...) of the sample results in a background signal. A fit to the experimental data taking into account the detector response is shown in fig. 7. Reasonable agreement is obtained assuming a 1.1 ps pump pulse and a distance of the sample from the center of the ring of 1.5 $\mathrm{mm}$. The background obtained for negative delay is well fit by assuming that the ring misalignment is equivalent to a beam splitter reflectivity of $40 \%$. The pulse duration obtained in the fit is longer than the experimental one. We believe that the rather long rising edges of the gate comes from the fact that the sample was $2 \mathrm{~mm}$ thick which introduces propagation effects. In order to obtain the ultimate time resolution the interaction region thickness must be kept on the order of the pump pulse spatial extension $c t_{p}$ by either reducing the sample thickness or increasing the angle between the pump and signal beams. We want to emphasize the fact that even in this preliminary experiment the beam diameters on the sample were several millimeters which means that this technique can easily be used to gate $2 \mathrm{D}$ images.

In summary, we have shown that Sagnac interferometers can be used as ultrafast gates. This tech- 
nique leads to very high contrast, high transmission optical switches and can even provide amplification of the gated signal. In a feasibility experiment we used a solid-state saturable absorber and obtained a picosecond gate. More experiments need to be done to understand the limitation of such a technique. For example, it is not clear whether the fluorescence of an amplifier placed in an interferometer will lead to a background in the gated signal. This technique can be associated with other spectroscopy [1-3] or gating techniques [4-9] since the signal leaking from a Sagnac interferometer is at the same wavelength and is coherent with the initial signal even after gating and amplification. In particular one can use such a gate as a front end of a holographic gate system [6], increasing by several orders of magnitude the contrast of the hologram. Finally we want to note that a Sagnac interferometer can easily be miniaturized and integrated in a semiconductor device.

This research was partially supported by the National Science Foundation through the Center for Ultrafast Optical Science under STC PHY 8920108. Jeff Squier acknowledges the support of a fellowship from Allied Signal Corporation. François Salin is on leave from Institut d'Optique Théorique et Appliquée, Unité Associée au Centre National de la Recherche Scientifique (France).

\section{References}

[1] D. Hulin, J. Etchepare, A. Antonetti, L.L. Chase, G. Grillon, A. Migus and A. Myserowicz, Appl. Phys. Lett. 45 (1984) 993.

[2] D. Hulin, A. Migus, A. Antonetti, I. Ledoux, J. Badau, J.L. Oudar and J. Zyss, Appl. Phys. Lett. 49 (1986) 761.

[3] J. Shah, IEEE J. Quantum Electron. 24 (1988) 276.

[4] M.A. Duguay and A.T. Mattick, Appl. Optics 10 (1971) 2162.

[5] M.C. Downer, R.L. Fork and C.V. Shank, J. Opt. Soc. Am. B2 (1985) 595 .

[6] H. Chen, Y. Chen, D. Dilworth, E. Leith, J. Lopez and J. Valdmanis, Optics Lett. 16 (1991) 487.

[7] J.L. Martin, Y. Lecarpentier, A. Antonetti and G. Grillon, Med. Biol. Eng. Comput. 18 (1980) 250.

[8] See for example, Ultrashort light pulses, ed. S.L. Shapiro (Springer, Berlin, 1977) Ch. 3.

[9] J. Hebling, J. Kuhl and J. Kleniczki, J. Opt. Soc. Am. B 8 (1991) 1089.

[10] G. Sagnac, Radium 8 (1911) 241.

[11] A.E. Siegman, IEEE J. Quantum Electron. QE-29 (1973) 247.

[12] C. Bordé, G. Camy, B. Decomps and L. Pottier, Colloq. Int. CNRS 217 (1973) 231.

[13] T.F. Carruthers and I.N. Duling III, Optics Lett. 15 (1990) 804.

[14] R. Trutna and A.E. Siegman, IEEE J. Quantum Electron. QE-13 (1977) 955.

[15] Y. Li, G. Eichmann and R.R. Alfano, Appl. Optics 25 (1986) 209.

[16] R. Trebino and C.C. Hayden, Optics Lett. 16 (1991) 493.

[17] J. Squier, F. Salin, G. Mourou and D. Harter, Optics Lett. 16 (1991) 324. 